\title{
ARQUITETURA E DESENVOLVIMENTO DE UM SOFTWARE EDUCATIVO PARA O ENSINO DE PROBABILIDADE E ESTATÍSTICA
}

\author{
Rafael Dornelles Lima ${ }^{1}$, Cristiane de Fatima Budek Dias ${ }^{2}$, Guataçara dos Santos Junior ${ }^{2}$, \\ Cristina Maria Mesquita Gomes ${ }^{3}$ e Rui Pedro Sanches de Castro Lopes ${ }^{3}$ \\ ${ }^{1}$ Faculdade Educacional da Lapa - FAEL, Brasil \\ ${ }^{2}$ Universidade Tecnológica Federal do Paraná - UTFPR, Brasil \\ ${ }^{3}$ Instituto Politécnico de Bragança - IPB, Portugal
}

\begin{abstract}
RESUMO
O presente artigo tem como objetivo apresentar a arquitetura de um Ambiente Virtual de Aprendizagem para o ensino de Probabilidade e Estatística, o Stat1, desenvolvido em uma pesquisa de Mestrado em Ensino de Ciência e Tecnologia da Universidade Tecnológica Federal do Paraná - UTFPR, Brasil. O Stat1 foi desenvolvido com aporte na metodologia Ágil, com enfoque em alguns artefatos do framework Scrum e a arquitetura seguiu o modelo REST (Representational State Transfer). Com isso, foi possível que os professores participassem ativamente do processo de construção e que o ambiente tenha possibilidades de complementação e melhorias, as quais poderão ser realizadas de acordo com as necessidades dos usuários e dos pesquisadores da Educação Estatística, ou de outras linhas de pesquisa e áreas do conhecimento. O Stat1 contempla as ferramentas: Conceitos; Pesquisa; Blog; Plano de aula; Leitura e Fórum. Tais ferramentas podem constituir-se em espaços para a interação entre professores e alunos; alunos e alunos; professores e professores; entre escolas; e entre escola e sociedade.
\end{abstract}

\section{PALAVRAS-CHAVE}

Software Educativo, Arquitetura, Stat1

\section{INTRODUÇÃO}

O desenvolvimento de um software educativo se dá com o propósito de conduzir o aluno na construção de conhecimentos sobre determinado conteúdo didático (Lacerda, 2007). Algumas características são fundamentais em softwares educativos: a presença de uma fundamentação pedagógica atrelada a todo seu desenvolvimento; uma finalidade didática, que tem a intenção de possibilitar a construção do conhecimento pelo aluno, de acordo com o currículo escolar; a permissão de interação entre o aluno e o software, que tende a ser mediada pelo professor; a facilidade de uso, pois é fundamental que se permita a qualquer usuário a capacidade de desenvolver atividades no software, sem a necessidade de conhecimentos computacionais avançados (Lacerda, 2007).

Assim, subentende-se que, desenvolver softwares educativos, é uma tarefa que exige ações e articulações entre conteúdos curriculares, metodologias educativas e práticas pedagógicas eficientes para que o software seja adequado às necessidades do processo de ensino e aprendizagem. O processo de desenvolvimento de softwares educativos precisa envolver, tanto as questões técnicas de funcionamento do sistema, quanto os aspectos didáticos e pedagógicos que constituem a base do processo de ensino e de aprendizagem (Lacerda, 2007). Ou seja, é preciso levar em conta que, no processo educativo, "uma boa parte do processo não é informatizável, pois acontece no cérebro da criança e/ou do professor" (Lacerda, 2007), considerando-se que cada sujeito é singular e requer atenção.

Neste artigo, tem-se como objetivo apresentar a arquitetura de um Ambiente Virtual de Aprendizagem (AVA) para o ensino de Probabilidade e Estatística, o Stat1, o qual foi desenvolvido com aporte no currículo escolar brasileiro e com a participação de professores em exercício. 


\section{STAT1: PROCESSO DE ELICITAÇÃO DE REQUISITOS}

O Stat1 é um Ambiente Virtual de Aprendizagem para o ensino de Probabilidade e Estatística que foi desenvolvido em uma pesquisa de Mestrado em Ensino de Ciência e Tecnologia, da Universidade Tecnológica Federal do Paraná - UTFPR/Brasil. Tal ambiente foi desenvolvido por uma equipe multidisciplinar: profissionais da área da educação, matemáticos e desenvolvedores de sistemas. O delineamento das propostas se deu com base nos documentos curriculares oficiais (Parâmetros Curriculares Nacionais (Brasil, 1997), Diretrizes Curriculares Nacionais (Brasil, 2013) e municipais (Ponta Grossa, 2015)) e nas práticas docentes, analisadas em uma pesquisa aplicada com professores dos anos iniciais do Ensino Fundamental de uma cidade do estado do Paraná/Brasil.

O Stat1 teve como aporte metodológico de desenvolvimento, a metodologia Ágil, mais especificamente o Scrum, o qual permite manter o desenvolvimento de software sempre ativo. O Scrum possui artefatos próprios de desenvolvimento e segue algumas etapas desde o início do projeto até sua finalização. No projeto do Stat1 seguiu-se apenas alguns dos artefatos e das etapas, como o levantamento de requisitos; a criação do Backlog de Produto e o Planejamento de Releases.

Para o levantamento de requisitos foi realizada a leitura dos documentos curriculares oficiais e foi aplicado um questionário piloto a 37 professores, que buscou levantar o que eles trabalhavam de Probabilidade e Estatística em suas aulas e como faziam isso (Dias, Pereira e Santos Junior, 2019). Com isso foram desenvolvidas as primeiras propostas para o AVA, ou seja, do protótipo do ambiente. Esse protótipo foi apresentado a 17 professores, em um encontro de coparticipação no desenvolvimento, no qual, foram coletadas as histórias de usuário, para o levantamento dos requisitos e geração do Backlog de Produto.

Cada professor, participante do encontro, recebeu um cartão (Figuras 1 e 2), no qual deveria descrever qual funcionalidade desejava para cada ferramenta do AVA e o porquê de tal solicitação (histórias de usuário). Algumas dificuldades foram notadas nas descrições dessas histórias, pois, alguns professores demonstraram receio e não conseguiram exprimir com clareza seus anseios e os motivos pelos quais solicitavam determinadas funcionalidades para o AVA (Figura 2). Esse fato pode ter ocorrido pela pouca familiaridade dos professores com projetos de desenvolvimento de softwares educativos (Dias, 2016) ou com softwares, em geral.

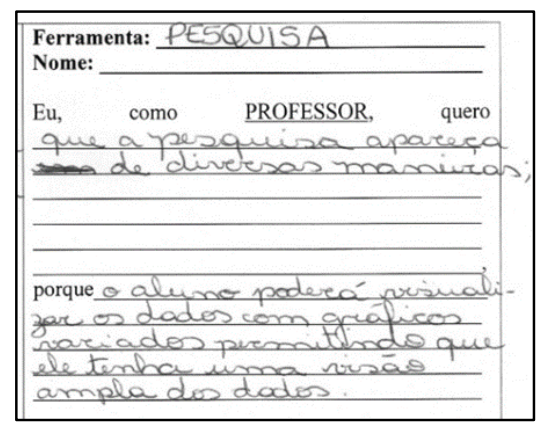

Figura 1. Exemplo de história de usuário Fonte: da pesquisa, 2015

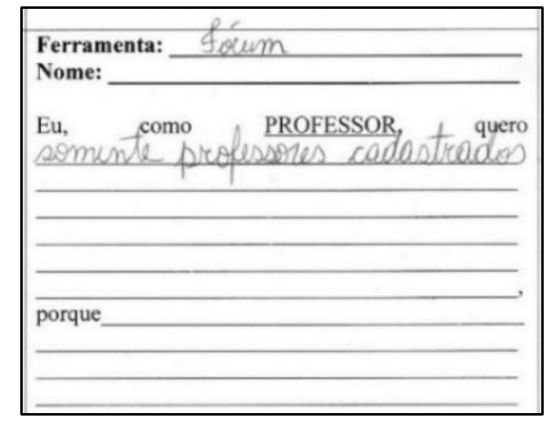

Figura 2. Exemplo de história de usuário sem definição de motivo Fonte: da pesquisa, 2015

Nota-se, na Figura 2, que o professor(a) solicita uma funcionalidade específica para uma das ferramentas do AVA, no entanto, não define o porquê de tal solicitação. Em muitos outros cartões, também, não há a especificação do porquê das solicitações, isso demonstra que, talvez, essa funcionalidade não seja tão necessária, já que não há uma justificativa de sua existência no sistema.

De acordo com o Backlog de Produto gerado, o Stat1 foi desenvolvido com as funcionalidades especificadas no diagrama de caso de uso apresentado na Figura 3. 


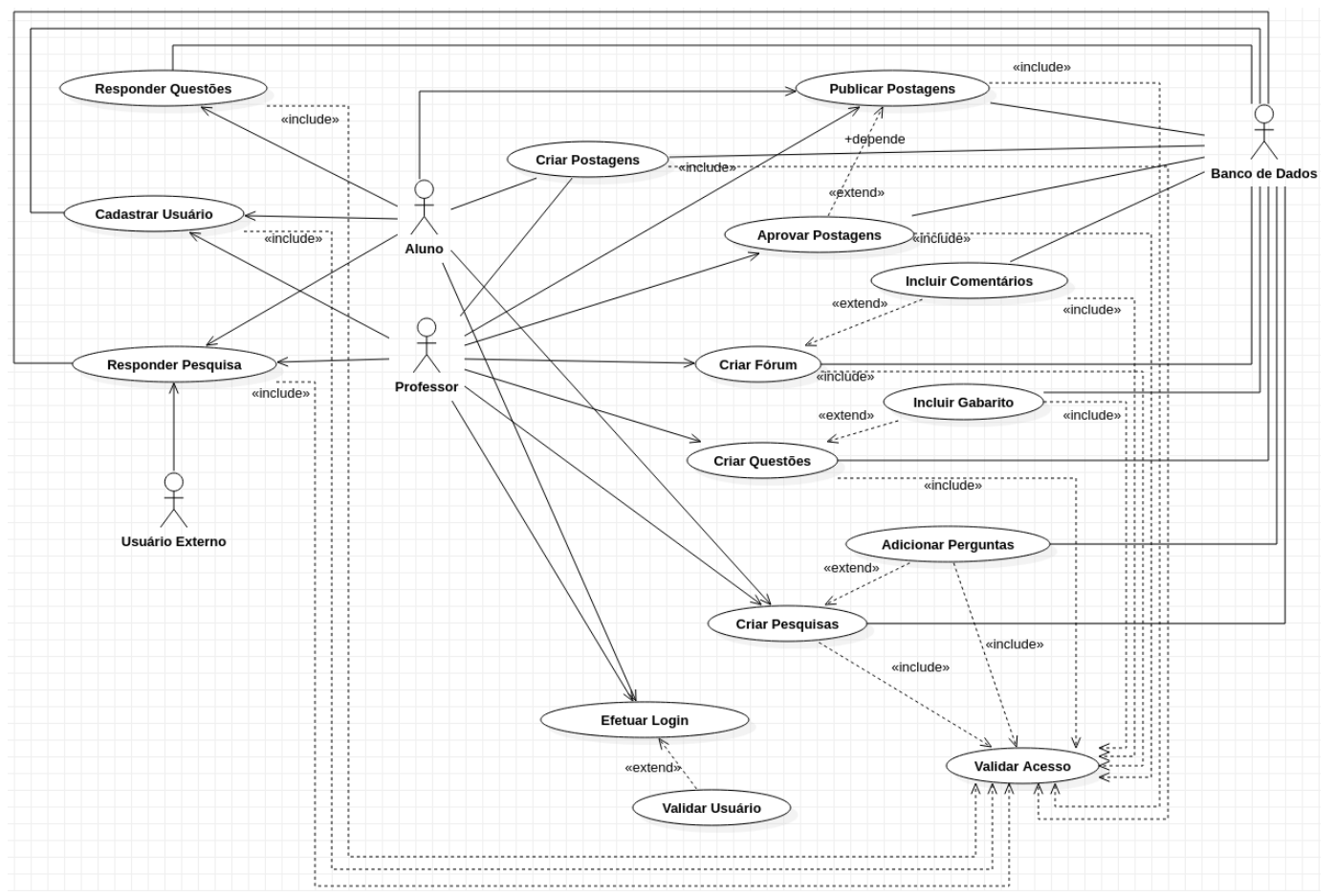

Figura 3. Diagrama de Caso de Uso do Stat1

Fonte: elaborado pelos autores

Como se observa no diagrama, a interação no Stat1 está projetada de acordo com três perfis de atores: aluno, professor e usuário externo. O Stat1 tem seis ferramentas: Conceitos; Pesquisa; Blog; Plano de Aula; Leitura e Fórum. Conceitos é um espaço destinado aos conceitos referentes à Probabilidade e Estatística. Tal ferramenta foi idealizada de forma que alunos, professores e usuários externos tenham acesso a conceitos e possam entender melhor as questões referentes à Probabilidade e Estatística.

A Pesquisa possibilita a interação com as etapas de uma investigação Estatística. Permite que professores e alunos construam um instrumento de pesquisa e que coletem dados a partir do mesmo. Ainda, é possível responder a pesquisas e visualizar as já realizadas. Usuários externos têm acesso a essa ferramenta e podem responder às pesquisas em andamento. $\mathrm{O}$ usuário cadastrado como professor pode responder; cadastrar; publicar e avaliar uma pesquisa (obter os resultados após as respostas dos demais usuários). $\mathrm{O}$ aluno poderá responder a uma pesquisa em andamento, cadastrar; enviar para a publicação e avaliar uma pesquisa. Ao enviar para a publicação o professor receberá essa pesquisa, avaliará e, caso haja necessidade de alterações, retornará ao aluno para as correções necessárias.

O Blog é um espaço de compartilhamento de informações a respeito das investigações estatísticas realizadas na turma. $\mathrm{O}$ intuito é de que sejam realizadas análises sobre os dados coletados e de que a turma possa descrever os dados e as conclusões, utilizando-se das terminologias probabilísticas e estatísticas adequadas. O usuário externo terá acesso aos textos postados no Blog, entretanto, somente usuários cadastrados poderão escrever tais textos. A publicação fica restrita aos professores, ou seja, os posts criados pelos alunos serão encaminhados para o professor, o qual fará a leitura e observará a necessidade de correção. Após isso, retornará ao aluno para que ele faça as alterações sugeridas e possa submetê-lo novamente para a publicação.

Plano de Aula é um espaço para a divulgação de planos de aula que se utilizem do Stat1, ou não, para o ensino de Probabilidade e Estatística. É uma proposta para que os professores compartilhem suas ideias e práticas. Usuários externos, alunos e professores poderão visualizar os planos de aula, entretanto, somente o usuário com o perfil de professor poderá cadastrar e publicar um plano de aula.

Leitura constitui-se em um espaço com textos e links de referências externas que tratam dos conteúdos de Probabilidade e Estatística: artigos, pesquisas, vídeos, entre outros. Essa ferramenta foi pensada com o intuito de auxiliar o professor na busca por sua própria formação, considerando que a leitura pode trazer conhecimentos importantes para o ensino de Probabilidade e Estatística. O acesso e gerenciamento da 
ferramenta se dará apenas para usuários cadastrados como professores, ou seja, somente professores poderão indicar textos neste espaço.

O Fórum é semelhante aos fóruns comumente encontrados em ambientes virtuais. Composta de um espaço que possibilita a postagem de dúvidas e a troca de experiências entre os docentes. O acesso e o gerenciamento dessa ferramenta são possibilitados aos usuários cadastrados como professores, que terão a oportunidade de incluir temas de discussão e comentar as postagens dos colegas. O professor poderá cadastrar tópicos de discussão para o fórum e poderá comentar e discutir sobre os temas propostos por outros professores.

\subsection{Arquitetura}

Para que o Stat1 possa ser acessado em diferentes plataformas e para facilitar o desenvolvimento futuro de outras áreas, ou de reutilização de algumas de suas partes para o desenvolvimento de outras ferramentas, utilizou-se o modelo arquitetural REST (Representational State Transfer) (Fielding, 2000). A Figura 4 apresenta o modelo arquitetural do Stat1.

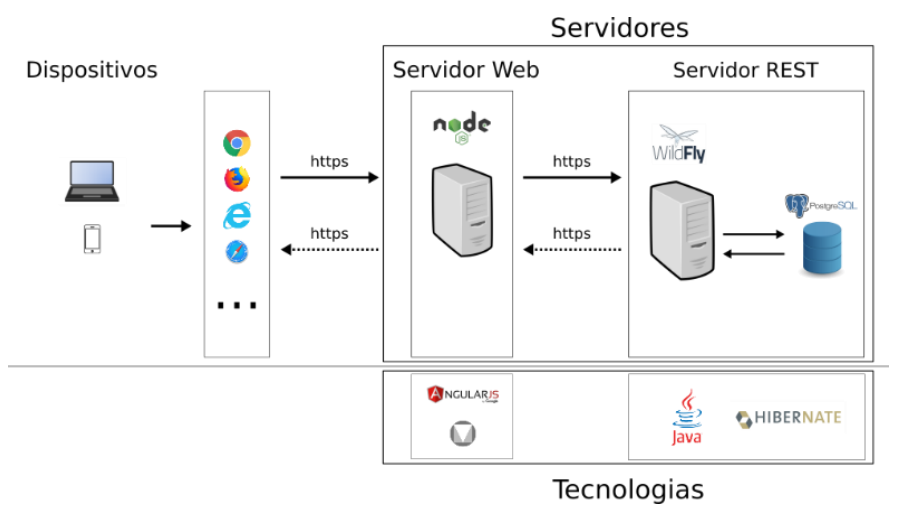

Figura 4. Arquitetura do Stat1

Fonte: elaborada pelos autores

Como apresentado na Figura 4, o usuário acessa a interface do Stat1 utilizando um navegador de Internet. O front-end do ambiente está hospedado em um servidor web NodeJs, que por sua vez executa requisições REST ao servidor back-end WildFly, o qual consulta os dados no banco de dados PostgreSQL.

Para a representação dos dados utilizou-se JSON (JavaScript Object Notation), embora seja possível a transmissão em outros formatos. Com o formato de arquivo JSON é possível estruturar os dados de forma mais compacta que em formatos como XML. Como se trata de um software educativo, este aspecto é extremamente importante, pois é preciso considerar o acesso à rede das escolas brasileiras, que nem sempre contam com uma banda de boa velocidade.

As demais tecnologias utilizadas no desenvolvimento do Stat1 foram: Java Enterprise Edition (JEE 8), AngularJS, Material Design, Hibernate. Como a equipe de desenvolvimento do Stat1, contou apenas com um desenvolvedor com expertise na tecnologia Java, isso possibilitou maior produtividade em menor tempo. Além disso, aproveitou-se da estabilidade, suporte da comunidade e robustez dessa tecnologia. Optou-se pelo AngularJS, por possuir uma curva de aprendizado relativamente baixa e, considerando a facilidade para manipulação das páginas Web, consumo dos serviços REST e por proporcionar maior interatividade ao usuário, definiu-se o desenvolvimento da interface do usuário com essa tecnologia. O Material Design foi escolhido por oportunizar melhor experiência ao usuário, aproveitando o know-how dos engenheiros do Google, criadores desse conceito de design. Além disso, é facilmente configurado com o AngularJs. Já o Hibernate ORM, foi usado para deixar o Stat1 mais flexível possível e para facilitar sua manutenção.

Para armazenamento dos dados, optou-se pela utilização do banco de dados PostgreSQL, pelo fato de ser um poderoso sistema de banco de dados objeto-relacional de código-fonte aberto. O servidor Wildfly foi utilizado, para a execução do back-end, pela compatibilidade com a tecnologia JEE e por ser um servidor Web que proporciona inicialização rápida, desempenho e escalabilidade, facilidade de administração, de 
código aberto (open source). E, para executar o ambiente front-end do Stat1, optou-se pelo Node.js, por ser um servidor leve, de código aberto e de fácil implantação e escalabilidade.

Por se tratar de um ambiente público e com poucos recursos a serem investidos, buscou-se utilizar tecnologias gratuitas ou open source para o desenvolvimento e disponibilização do Stat1. Vale ressaltar que, embora sejam gratuitas e de código aberto, são tecnologias muito utilizadas no mercado, inclusive por grandes empresas e administrações públicas.

\section{CONCLUSÃO}

Com as tecnologias utilizadas na criação do Stat1, foi possível desenvolver um ambiente virtual com possibilidades de complementação e melhorias. As complementações poderão ser realizadas de acordo com as necessidades dos usuários e dos pesquisadores da Educação Estatística, integrantes, ou não, do grupo de pesquisa no qual se originaram as primeiras ideias sobre suas funcionalidades. Além disso, há possibilidades de reutilização de partes do ambiente na construção de propostas para outros conteúdos e disciplinas curriculares.

O intuito foi de construir um ambiente que, apesar de, neste momento estar disponível para acesso apenas em navegadores Web, no futuro, seja possível evoluir sua estrutura com a criação de novos serviços, ou, ainda, que permita o acesso por meio de dispositivos móveis, com desenvolvimento nativo para a respectiva plataforma, consumindo os mesmos serviços. Isso, também, facilita a continuidade do trabalho dentro da comunidade de pesquisa, pois o Stat1 pode agregar novas ferramentas para se adequar a outros conteúdos e linhas de estudo. As ferramentas do Stat1 permitem acesso e gerenciamento por diferentes atores e se oportuniza a interação entre eles.

Mais informações sobre o Stat1 podem ser obtidas em: http://repositorio.utfpr.edu.br/jspui/handle/1/1873 e em http://dx.doi.org/10.5965/2357724X06112018262.

\section{REFERÊNCIAS}

AngularJS, 2019. Architecture overview. https://angularjs.org/

Brasil, 1997. Parâmetros Curriculares Nacionais: Matemática, $1^{\circ}$ e $2^{\circ}$ ciclos do ensino fundamental. Ministério da Educação. Secretaria do Ensino Fundamental. Brasília, DF.

Brasil, 2013. Diretrizes Curriculares Nacionais Gerais da Educação Básica. Ministério da Educação. Secretaria de Educação Básica. Diretoria de Currículos e Educação Integral. Brasília: MEC, SEB, DICEI.

Dias, C. de F.B., Junior, G. dos S., and Lima, R.D., 2018. Produto educacional para o ensino de Probabilidade e Estatística: Stat1. Boletin Online de Educação Matemática (BoEM). Vol. 6, No. 11, pp. 262-281.

Dias, C. F. B., 2016. Ambiente virtual de aprendizagem para o ensino de probabilidade e estatística nos anos iniciais do ensino fundamental. Dissertação de Mestrado em Ensino de Ciência e Tecnologia, Universidade Tecnológica Federal do Paraná. Ponta Grossa.

Fielding, R. T., 2000. Architectural styles and the design of networked-based software architectures. Dissertação de Doutoramento Dept. of Information and Computer Science, University of California, Irvine.

Hibernate ORM, 2019. Your relational data. Objectively. http://hibernate.org/orm/

Lacerda, R. de A., 2007. Proposta de um modelo para análise de requisitos de software educativo. Dissertação de Mestrado em Educação, Universidade de Brasília, UNB, Brasília/Distrito Federal.

Material Designer, 2019. Introdution. https://material.io/design/introduction

Node.js, 2019. About Node.js. https://nodejs.org/en/about/

Oracle, 2019. Java ${ }^{\mathrm{TM}} \mathrm{EE}$ at a Glance: https://www.oracle.com/technetwork/pt/java/javaee/overview/index.html

Ponta Grossa, 2015. Diretrizes curriculares: ensino fundamental. Prefeitura Municipal de Ponta Grossa, Secretaria Municipal de Educação. Ponta Grossa, Brasil.

PostgreSQL, 2019. About. https://www.postgresql.org/about/

WildFly, 2019. What is WildFly? https://wildfly.org/about/ 